\title{
Memória Coletiva e Objetos Biográficos: estudo dos oratórios em Minas Gerais/Brasil do período colonial ${ }^{1}$
}

\author{
Memória coletiva y objetos biográficos: estudo dos oratórios em Minas \\ Gerais/Brasil del período colonial
}

\author{
Collective Memory and Biographical Objects: study of oratories in Minas \\ Gerais / Brazil from the colonial period
}

Nadja Maria Mourão ${ }^{2}$

Ana Célia Carneiro Oliveira ${ }^{3}$

\begin{abstract}
Resumo
O cotidiano da sociedade emerge das tradições de um povo e do seu convívio com o entorno. Alguns objetos recebem uma carga emotiva da vida humana e podem se transformar em objetos biográficos - que representam a cultura de uma sociedade. A memória coletiva é o conjunto de fatos escolhidos por um grupo social, com significados que constituem sua identidade, seus hábitos, sua cultura e tradições. Este trabalho apresenta o estudo dos oratórios, objetos biográficos oriundos da cultura portuguesa, identificados pela memória coletiva, de comunidades do Estado de Minas Gerais, desde o período colonial. A metodologia se realiza pela pesquisa bibliográfica de contextos temáticos e históricos. Em definição, oratório é um nicho ou armário, como uma capela doméstica. Essa pequena capela surgiu na Idade Média, como local para orações e reflexões, para o rei dedicado às práticas religiosas. Os oratórios são objetos que se estabeleceram na cultura mineira, sendo encontrados desde os grandes casarões até em senzalas, desde os tempos do Brasil Colônia. Há registros destes altares nas famílias devotas, que tem o desejo de guardar as relíquias e os objetos de piedade, em atitudes de intimidade com o mundo do sagrado. Os oratórios eram passados de mãe para filha, que guardavam os objetos de devoção em seus quartos e, quando se casavam, eles eram conduzidos para a nova família. Alguns oratórios são bem rebuscados, com entalhes, pinturas e diversos ornamentos. Outros oratórios são simples, como se fossem apenas pequenos armários. Os diversos materiais, tamanhos e estilos mostram hábitos e tradições. Além da influência da cultura portuguesa e seus artistas, são encontrados registros de entalhes de artistas espanhóis e franceses, que ajudaram a manter a herança da religiosidade nas famílias brasileiras. Contudo, esses objetos se constituem na diversidade da cultura mineira, oriundas da memória coletiva de seu povo.
\end{abstract}

Palavras chave: Memória coletiva; objetos bibliográficos; oratório; período colonial; Minas Gerais.

\footnotetext{
${ }^{1}$ Artigo apresentado no Simpósio Temático 03 - Repensar, Refletir, Interpretar e Reinterpretar a Memória, Iidentidade e o Patrimônio cultural da América Colonial, durante o II Seminário Latino-Americano de Estudos em Cultura - SEMLACult em Foz do Iguaçu/PR, Brasil, 2018.
}

${ }^{2}$ Mestra e Doutoranda em Design; CEDTec - Centro de Estudos em Design e Tecnologia da Escola de Design da Universidade do Estado de Minas Gerais/UEMG; Cidade de Belo Horizonte/ Estado de Minas Gerais/Brasil; nadjamourao@gmail.com

\footnotetext{
${ }^{3}$ Mestra em Design; CEDTec - Centro de Estudos em Design e Tecnologia da Escola de Design da Universidade do Estado de Minas Gerais/UEMG; Cidade de Belo Horizonte/ Estado de Minas Gerais/Brasil; anaceliadesign@gmail.com
} 


\begin{abstract}
Resumen
El cotidiano de la sociedad emerge de las tradiciones de un pueblo y de su convivencia con el entorno. Algunos objetos reciben una carga emotiva de la vida humana y pueden transformarse en objetos biográficos - que representan la cultura de una sociedad. La memoria colectiva es el conjunto de hechos elegidos por un grupo social, con significados que constituyen su identidad, sus hábitos, su cultura y tradiciones. En este trabajo se presenta el estudio de los oratorios, objetos biográficos procedentes de la cultura portuguesa, identificados por la memoria colectiva del Estado de Minas Gerais comunidades de la época colonial. La metodología se realiza por la investigación bibliográfica de contextos temáticos e históricos. En definición, oratorio es un nicho o armario, como una capilla doméstica. Esta pequeña capilla surgió en la Edad Media, como lugar para oraciones y reflexiones, para el rey dedicado a las prácticas religiosas. Los oratorios son objetos que se establecieron en la cultura minera, siendo encontrados desde los grandes caserones hasta en senzalas, desde los tiempos del Brasil Colonia. Hay registros de estos altares en las familias devotas, que tienen el deseo de guardar las reliquias y los objetos de piedad, en actitudes de intimidad con el mundo de lo sagrado. Los oratorios eran pasados de madre a hija, que guardaban los objetos de devoción en sus habitaciones y, cuando se casaban, eran conducidos a la nueva familia. Algunos oratorios son bien rebuscados, con muescas, pinturas y diversos ornamentos. Otros oratorios son simples, como si fueran sólo pequeños armarios. Los diversos materiales, tamaños y estilos muestran hábitos y tradiciones. Además de la influencia de la cultura portuguesa y sus artistas se encuentran artistas de España y Francia ranuras registros, lo que ayudó a mantener el patrimonio de la religión en las familias brasileñas. Sin embargo, esos objetos se constituyen en la diversidad de la cultura minera, oriundos de la memoria colectiva de su pueblo.
\end{abstract}

Palavras chave: Memória coletiva; objetos bibliográficos; la oratoria; período colonial; Minas Gerais.

\begin{abstract}
The daily life of society emerges from the traditions of a people and from their conviviality with the environment. Some objects are emotionally charged with human life and can become biographical objects representing the culture of a society. Collective memory is the set of facts chosen by a social group, with meanings that constitute their identity, their habits, their culture and traditions. This work presents the study of oratories, biographical objects originating from Portuguese culture, identified by the collective memory of communities in the State of Minas Gerais, since the colonial period. The methodology is carried out by the bibliographic research of thematic and historical contexts. In definition, oratory is a niche or closet, like a domestic chapel. This small chapel arose in the Middle Ages, as a place for prayers and reflections, for the king dedicated to religious practices. The oratories are objects that have settled down in the culture of Minas Gerais, being found from the great houses until in senzalas, since the times of the Brazil Colony. There are records of these altars in devout families, which have the desire to keep relics and objects of piety, in attitudes of intimacy with the sacred world. The oratories were passed from mother to daughter, who kept the objects of devotion in their rooms and, when they were married, they were taken to the new family. Some oratories are refined, with carvings, paintings and various ornaments. Other oratories are simple, as if they were just small cabinets. The various materials, sizes and styles show habits and traditions. In addition to the influence of Portuguese culture and its artists, there are records of carvings of Spanish and French artists, which helped to maintain the religiosity heritage in Brazilian families. However, these objects constitute the diversity of the culture of Minas Gerais, originating from the collective memory of its people.
\end{abstract}

Keywords: Collective memory; bibliographic objects; oratory; colonial period; Minas Gerais.

\title{
1. Introdução
}

A convivência social e relações com o entorno geram os costumes, as tradições e a história de um povo. O processo de valorização do território se realiza no campo de dois circuitos: um - social, e o outro - natural, conforme Santos (2007). Considera-se o circuito social quando o território é analisado a partir do momento em que é pensado juntamente com 
os atores que o utilizam. Já o circuito natural se estabelece a partir das características físicas geográficas. $\mathrm{O}$ autor esclarece que o ser humano necessita de ambas as relações para garantir sua existência.

O território tem que ser entendido como o território usado, não o território em si. O território usado é o chão mais a identidade. A identidade é o sentimento de pertencer àquilo que nos pertence. $\mathrm{O}$ território é o fundamento do trabalho; o lugar da residência, das trocas materiais e espirituais e do exercício da vida (SANTOS, 2007, p. 22).

Segundo Le Betron (2009) o homem está afetivamente presente no mundo, tanto nas relações com os outros, quanto com os objetos que o circundam. O produto se torna afetivo a partir do momento que ele adquire importância para o usuário, ao ponto dele desenvolver relações afetivas com o objeto, independentemente da sua natureza, ou seja, a afetividade trata de relações.

Para o ser humano, são os padrões mentais que geram as sensações, efeitos emocionais que se refletem nas relações como os objetos. "O cérebro humano mapeia qualquer objeto que esteja fora dele, qualquer ação que ocorra fora dele e todas as relações que os objetos e as ações assumem no tempo e no espaço, corpo, cérebro e mente" (DAMÁSIO, 2011, p.88). Dessa forma, o corpo humano é a fronteira entre os sinais do ambiente e a percepção do cérebro, que mapeia e transforma em sinais essas relações.

Alguns objetos recebem uma carga emotiva da vida humana e podem se transformar em objetos biográficos, elementos transcendentes da cultura de uma sociedade. É possível observar a transformação oriunda da apropriação individual de um objeto, pela análise de seu uso, como: uma xícara, um par de sapatos, um violão ou um anel, entre tantos outros. Estes passam a ser denominados objetos biográficos por carregarem significados que recordam pessoas ou momentos, contextualizando a história de vida de cada indivíduo, conforme Bosi (1979).

Compreende-se que os envolvimentos afetivos se referem a conhecimentos subjetivos, que revelam a forma como cada ser humano "[...] é afetado pelos acontecimentos da vida ou, melhor, pelo sentido que tais acontecimentos têm para ele" (PINO, 2000, p. 128). Portanto, pondera-se que a afetividade é a capacidade que os indivíduos têm de serem positiva ou negativamente envolvidos, com maior ou menor intensidade, por um dado artefato ou lugar, de forma que cada um deles situa um tipo de afinidade afetiva com esse artefato ou lugar e lhe atribui um sentido único. 
Este trabalho apresenta um breve estudo de oratórios, objetos biográficos oriundos da cultura portuguesa, identificados pela memória coletiva, de comunidades pertencentes ao Estado de Minas Gerais, desde o período colonial.

A metodologia se realiza pela pesquisa bibliográfica de contextos temáticos e históricos. Buscam-se bases conceituais para as temáticas de Memória Coletiva, Objetos Bibliográficos e Oratórios Mineiros, de forma a atender os procedimentos da pesquisa. “Como limitação do método proposto pode ser levantada a necessidade de o pesquisador ter conhecimento prévio sobre o tema de pesquisa. Durante todo o processo é necessária a intervenção do autor para identificação das palavras-chave e realização das filtragens" (TREINTA et al., 2012, p. 4). Dessa forma, são pesquisadas as denominações e o contexto no território em que se relacionam.

\section{Memória Coletiva e Objetos Bibliográficos}

A memória coletiva, de acordo com os pensadores Maurice Halbwachs, Michael Pollak e Beatriz Sarlo, é o conjunto de fatos escolhidos por um grupo social, com significados que constituem sua identidade, seus hábitos, cultura e tradições (Rios, 2014).

Para Rios (2014); Halbwach (2013), a memória coletiva é construída pelo convívio social entre as pessoas, considerando que a lembrança necessita de uma comunidade afetiva. Para tomar consistência, pode-se considerar que as lembranças de outros indivíduos que compõem o mesmo grupo no qual estamos inseridos, podem compor a percepção individual dos acontecimentos.

Pollak e Halbwachs esclarecem que a memória se faz na construção do passado em tempos atuais. A memória coletiva é variável, e também múltipla, pois cada grupo cultiva um conjunto particular de recordações, de acordo com Rios (2014). O autor ainda contempla os estudos de Beatriz Sarlo pela subjetividade da memória coletiva, pois é mais fácil lembrar um contexto do que a ação "o que lembrar". Para Sarlo, o indivíduo constrói a memória pelas diversas influências recebidas do meio externo.

Os objetos bibliográficos, conforme García (2009) constitui-se por obras, textos, traduções e edições. Mas, também são os objetos que carregam contextos históricos coletivos ou individuais, significativos entre gerações.

Há uma distinção entre os objetos biográficos e protocolares. Os protocolares seriam aqueles que fazem parte da vida das pessoas sem causar impacto, podem ser obsoletos. "Em relação aos biográficos, são todos aqueles que deixam marcas, tanto no âmbito pessoal como 
no social". Neste caso, surge a questão de preservação de tais objetos que pertenceram a pessoas já desaparecidas (SILVA, 2016, P.127).

$\mathrm{Na}$ relação com a pesquisa verifica-se que os oratórios são exemplos de objetos bibliográficos pela representatividade na vida das pessoas, de acordo com Russo (2010):

E assim, se contempla uma conformação especial na convocação de imagens, com destaque aos santos patronos e aos símbolos religiosos mais populares, representando relatos históricos ou de tradições religiosas associadas aos santos, como a constituição de cenas da vida de Cristo, da Virgem Maria e dos Santos do hagiológico cristão que exemplificam, entre outros, a caridade, a piedade, o sacrifício, a fé inabalável e a missão evangelizadora (RUSSO, 2010, p. 259).

Contudo, entre uns e outros, citamos os oratórios Algibeira ou de Viagem Miniatura, que são aqueles pequeninos oratórios que podem ser carregados nos bolsos, junto ao corpo do fiel. Como um sinal de proteção cotidiana pedido ao santo de devoção, dentro de pequenos invólucros. Estes, provavelmente se tornaram objetos de memória, contemplados quase que exclusivamente pelos usuários. Dessa forma, conforme Kubler (1977) deve-se observar os objetos além dos traços artísticos, mas também pelos aspectos simbólicos.

\section{Os Oratórios de Minas Gerais}

Oratório é um nicho ou armário, como uma capela doméstica. Essa pequena capela surgiu na Idade Média, como local para orações e reflexões, para o rei dedicado às práticas religiosas, conforme Russo (2011).

Nesse sentido, [...] "qualquer pessoa pode ter em casa um oratório, destinando para ele um local apropriado; resguardando em seu interior: quadros, estátuas, altar, etc., para meditar, rezar, fazer novena etc.”, sozinho ou com a família (RUSSO, 2011, p.1) apud (FERREIRA, 1916, p.9).

A expedição de Cabral, em 1500, transportou o primeiro oratório para o Brasil, com uma imagem de Nossa Senhora da Esperança, iniciando uma tradição religiosa neste país. Em Minas Gerais, há registros de oratórios desde os casarões do período colonial até os dias atuais, conforme Gutierrez (2014).

Em 1998 foi inaugurado o Museu do Oratório em Ouro Preto, Minas Gerais, com 162 oratórios e cerca de 300 imagens de santos, doados por Ângela Gutierrez ao IPHAN. Conforme site do Museu, os oratórios que compõem o acervo possuem, na grande maioria, à 
tradição barroca da criação religiosa, pelo fato de o período artístico corresponder à época de maior produção de arte e artesanato com finalidade religiosa no Brasil.

Gutierrez (2014) descrever que os oratórios eram passados de mãe para filha, que guardavam os objetos de devoção em seus quartos e, quando se casavam, eles eram conduzidos para a nova família. Colocados em altares nas residências de famílias devotas, que guardavam as relíquias e os objetos de piedade, em atitudes de intimidade com o mundo do sagrado.

Estes objetos de fé, com o sabor mágico e místico por diversas mãos, se difundiram no Brasil pelo processo de transportação cultural, inseridos pelo imaginário estético popular, oriundos de fontes portuguesas, desde o período colonial. Em Minas Gerais, a fé significava uma relação natural e imediata do cotidiano devocional com os santos, com a virgem Maria, e a santíssima Trindade. Os motivos do barroco mineiro se mantiveram ao longo dos anos e permanecem sob os trabalhos dos artesãos, conforme Ávila e Trindade (2014).

Alguns oratórios são bem rebuscados, com entalhes, pinturas e diversos ornamentos; outros são simples, como pequenos armários. É possível classificar os oratórios pelas funções e estilos: Os Oratórios de Viagem são chamados de Bala, pela forma semelhante ao projétil. Surgiram no noroeste brasileiro e no norte de Minas Gerais, transportados por tropeiros e mascates. Os Domiciliares eram encontrados em diversos ambientes da residência, refletindo o estilo de vida e as devoções da família. Para cada santo, uma devoção: Santo Antonio, para pedir o casamento das filhas; Santa Barbara, proteção dos que trabalhavam em minas; Santa Rita, para as causas impossíveis, e outros. Já os Oratórios Eruditos ou de Salão e de Sacristia ostentam formas refinadas e elegantes, destinados a espaços nobres sejam residenciais ou públicos (Gutierrez (2014).

A autora também relata a existência dos Afro-brasileiros com materiais diferenciados da madeira recortada e entalada ao ferro. Estão ligados, muitas vezes, aos rituais de cultos africanos ocultamente praticados na era colonial e imperial. Outras peças eram revestidas de Chinoiseries (estilo chinês) como ecos da influência oriental na cultura lusitana. Constatam-se também os Ermidas, que são grandes oratórios inseridos na arquitetura em locais de afluência pública. E, não menos importante, os Oratórios de Lapinha, típicos do final do século XVIII, populares em Minas Gerais, confeccionados com calcita, um mineral encontrado nas lapas de Minas Gerais. Alguns dos tipos citados estão representados na figura 1: Oratório de Bala (Icó, Ceará, séc. VIII), Oratório de Salão (Minas Gerais, séc. XVIII) e Oratório Lapinha (Minas Gerais, séc. XVIII). 

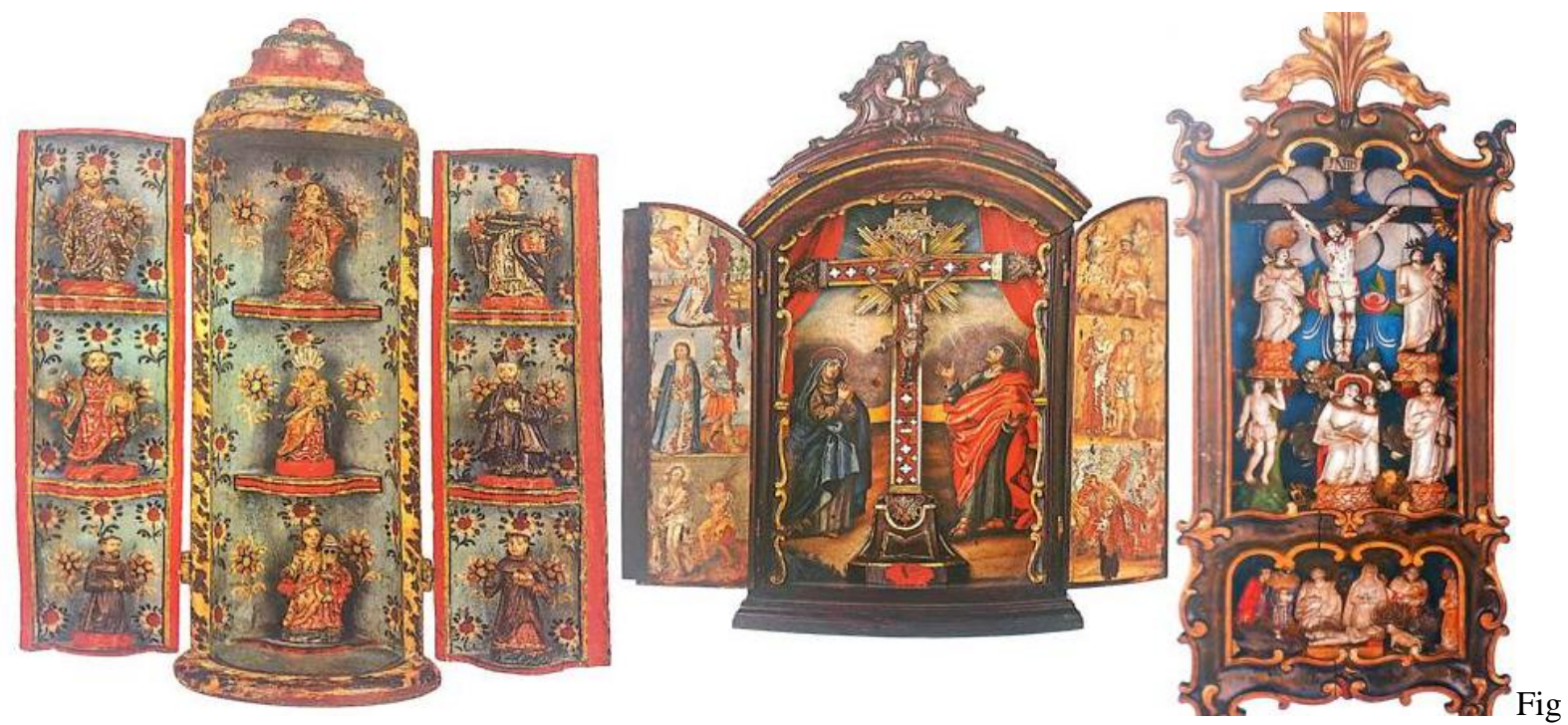

Figura 1: Oratório Bala, Oratório de Salão e Oratório Lapinha (da direita para esquerda)

Fonte: Catálogo do Museu do Oratório, 2014.

Os diversos materiais, tamanhos e estilos mostram hábitos e tradições. Além da influência da cultura portuguesa e seus artistas, são encontrados registros de entalhes de artistas espanhóis e franceses, que ajudaram a manter a herança da religiosidade nas famílias brasileiras, conforme Russo (2010).

\section{Influencias da religiosidade em artesãos de Minas Gerais}

As referências que o indivíduo adquire e que, por experiências vivenciadas, defini sua identificação com o mundo que o cerca. Como também, interfere nas ações no espaço onde está inserido, onde há influência do meio social, com as trocas e relações no contexto.

Entre os trabalhos desenvolvidos por artesãs, que conciliam o serviço doméstico com as práticas artesanais, estão os oratórios e Santo Antonio de buriti. O Santo Antonio de Buriti é um produto desenvolvido na comunidade de Serra das Araras, no Município de Chapada Gaúcha. A Associação dos Artesãos e Bordadeiras de Serra da Araras aperfeiçoa as técnicas de utilização dos "talos" ou braços de buritis, material amplamente utilizado na região. A Comunidade de Serra das Araras é uma das mais antigas na região, desde os tempos dos cangaceiros. Nas festas do santo padroeiro, Santo Antonio, a população aumenta significamente, nos três dias do festejo religioso, conforme Mourão (2011).

O Santo Antonio confeccionado de braço de buriti é muito frágil. Maria Rocicley, membro da associação, mostra na figura 2, como o santo pode ser comercializado em diversos 
tamanhos. Para o santo maior, há um modelo de oratório que também serve como embalagem para transporte.

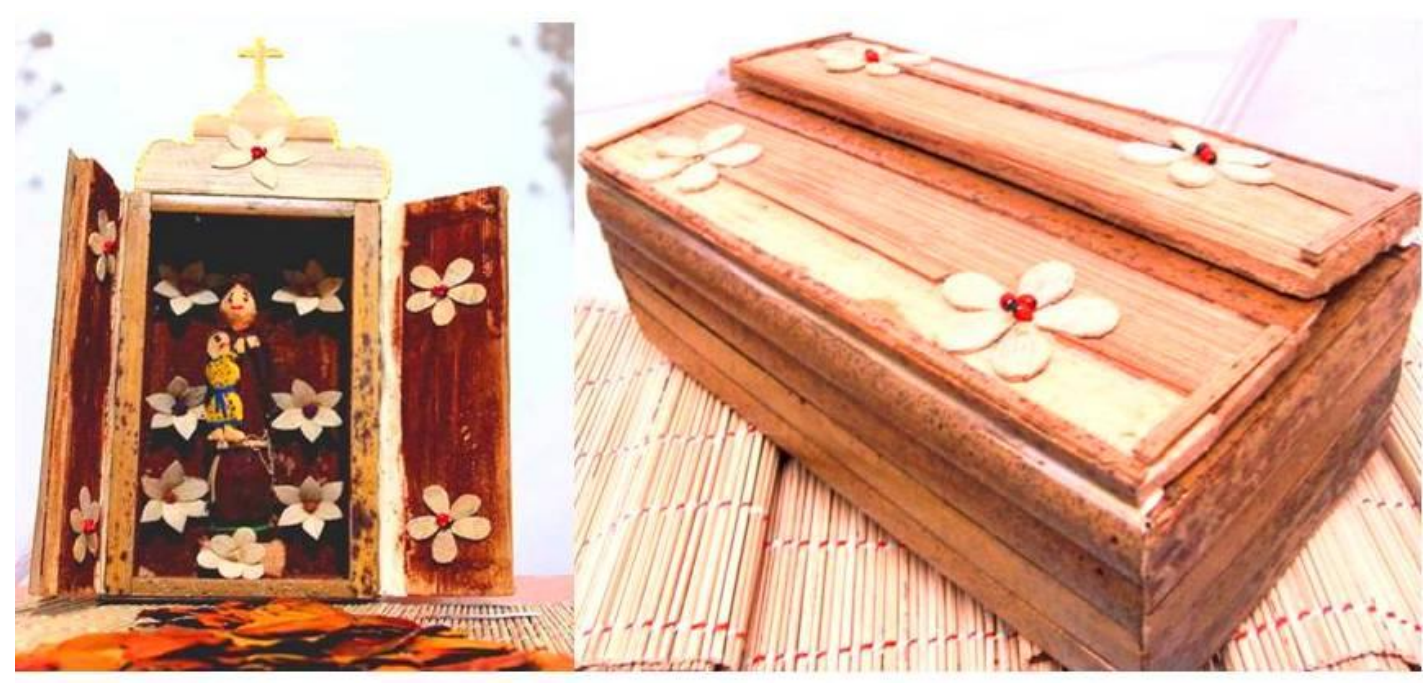

Figura 2: Oratório confeccionado de buriti da Associação de Serra das Araras/MG.

Fonte: Acervo da pesquisa, 2016.

Ainda sobre o trabalho do artesão religioso, Sennett (2012) amplia a reflexão acerca da cultura material para além do entendimento de que a construção das peças artesanais reflete, tão somente, as normas sociais, interesses econômicos, convicções religiosas e são capazes de contar e explicar a própria história da humanidade.

Assim, através da cultura material é possível aprender sobre a experiência humana em seu processo de habilidade artesanal, entendido como uma habilidade em constante busca da realização. No Brasil colonial, os trabalhos manuais estiveram conexos ao fazer feminino onde "obtinham exclusividade quando eram costureiras, doceiras, fiandeiras, criadas, cozinheiras ou lavadeiras, acabando por reproduzir os papéis que lhes eram dados tradicionalmente" (LAGES, 2007, p. 17).

Na figura 3, observam-se oratórios em materiais diversos, do artesanato popular, como também, os trabalhos de artesões do Centro Cape (Instituto Centro de Capacitação e Apoio ao Empreendedor), símbolos representativos da religiosidade e das relações afetivas dos artesãos com os santos confeccionados. 


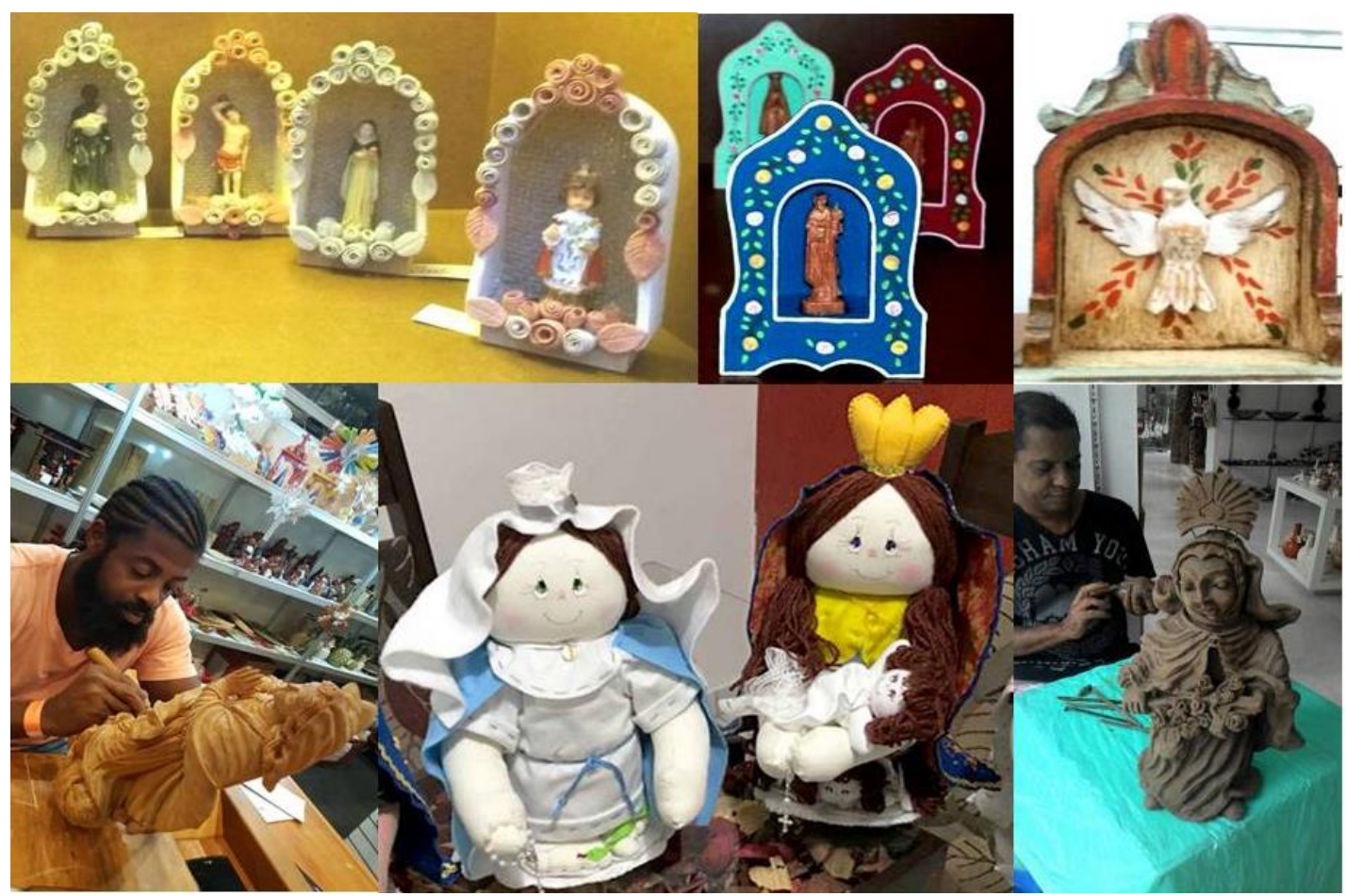

Figura 3: Oratórios em materiais diversos e os trabalhos de artesãos do Centro Cape.

Fonte: Acervo da pesquisa e Centro Cape, 2018.

Como atividade social e econômica, o artesanato traz um modelo diferenciado de competitividade, pois o diferencial de cada trabalho, principalmente o artesanato religioso, está nos traços culturais singulares de cada região, além da melhoria qualitativa de seus produtos. Para o artesão é também uma atividade de prazer e de desenvolvimento pessoal.

\section{Análise dos resultados}

Analisa-se a existência dos oratórios inseridos na formação da cultura brasileira a partir da colonização portuguesa. E a efervescência sociocultural estimulou a produção intensa dos mais variados tipos de oratórios, diferentes de outras culturas onde os santos também recebam seus oratórios para atos devocionais.

A cultura de uma sociedade é formada pela produção de bens e valores, que através das coordenadas de tempo e espaço caracterizam as identidades de seus membros, construindo memórias afetivas Dos exemplos mais simples aos eruditos, os oratórios passaram a ocupar espaços privilegiados, nas casas nos ambientes públicos, nas igrejas e até mesmo nas malas do povo mineiro. 
Os oratórios, como objetos bibliográficos assumiram os espaços em museus e principalmente, na concepção do Museu do Oratório em Minas Gerais, possibilitando as mais variadas formas de investigações. Estes objetos da memória coletiva contam relatos de hábitos e costumes e as tradições no território brasileiro, em espacial no território mineiro. Uma vez que, somente pela colação de inúmeros objetos foi possível conceber o museu.

\section{Considerações Finais}

Contudo, esses objetos se constituem na diversidade da cultura mineira, oriundas da memória coletiva de seu povo. Eles permanecem como representantes de uma forma de humanização do divino, assegurando a convivência intima do devoto com suas entidades tutelares.

A valorização dos saberes culturais, somados às memórias, geram experiências e emoções aplicáveis para a vida prática das novas gerações. A simples lembrança de um artefato ou lugar casual ou familiar é suficiente para fazer com que ele se eternize no coração do seu possuidor, como observado na conservação dos oratórios em residências. A relação afetiva construída ao longo do tempo entre as pessoas e artefatos é um fator aplicável na lembrança de pessoas com vínculo ao grupo social, construindo laços fortes de identidade.

Acredita-se que os oratórios, estabelecidos na história e como patrimônio cultural, pela diversidade de formas e materiais, se apresenta como produto consolidado no turismo de cidades religiosas. Os oratórios poderão gerar novas fronteiras, além do contexto religioso, que transcende os limites e apontam novas relações para a memória do povo.

\section{Referências}

AVILA, C; TRINDADE, S. C. A geografia do Sagrado na Minas Colonial. In: GUTIERREZ, A. (org.). Catálogo Oratório Relíquias do Barroco Brasileiro. Belo Horizonte: Instituto Cultural Flávio Gutierrez, 2014.

BOSI, E. O tempo vivo da memória: ensaios de psicologia social. São Paulo: Ateliê Editorial, 2003.

DAMÁSIO, A. E o cérebro criou o homem. São Paulo: Companhia das Letras, 2011.

GARCÍA, A. A. R. Los objetos bibliográficos confirmados en la integración compleja de la descripción y acceso a recursos. Revista Investigación Bibliotecológica, vol.23, $\mathrm{n}^{\mathrm{o}}$. 48, México: Centro Universitario de Investigaciones Bibliotecológicas de la UNAM, 2009. 
GUTIERREZ, A (Org.). Catálogo Oratório Relíquias do Barroco Brasileiro. Belo Horizonte: Instituto Cultural Flávio Gutierrez, 2014.

HALBWACHS, Maurice. A Memória Coletiva. Tradução de Beatriz Sidou. $2^{\mathrm{a}}$ ed. São Paulo: Ed. Centauro, 2013.

INSTITUTO CENTRO CAPE. Disponível em: 〈http://www.centrocape.org.br〉. Acesso em: 10 mai. 2019.

KUBLER, G. A forma do tempo: Observações sobre a História dos Objetos. Lisboa: Vega, $2^{\mathrm{a}}$. Edição, 1977.

LAGES, Sônia Regina Corrêa. Mulheres na encruzilhada: encontros e desencontros no discurso de mulheres possuídas pela entidade da Pomba-Gira Cigana na Umbanda. Tese de Doutorado. Rio de Janeiro: UERJ, 2007.

LE BETRON, D. As paixões ordinárias. Antropologia das emoções. Rio de Janeiro: Editora Vozes, 2009.

MOURÃO, Nadja Maria. Sustentabilidade na produção artesanal com resíduos vegetais: uma iniciativa prática de design sistêmico no cerrado mineiro. (Dissertação) Mestrado em Design. Belo Horizonte: Universidade do Estado de Minas Gerais, 2011.

MUSEU DO ORATÓRIO. Disponível em: 〈http://museudooratorio.org.br/>. Acesso em: 10 mai. 2019. .

PINO, Angel. A Afetividade e vida de relação. Campinas: UNICAMP, 2000.

RIOS, F. Memória coletiva e lembranças individuais a partir das perspectivas de Maurice Halbwachs, Michael Pollak e Beatriz Sarlo. In: Revista Intratextos, 2013, vol 5, no1, p. 1-22. DOI: <http://dx.doi.org/10.12957/intratextos.2013.7102>. Acesso em: 10 mai. 2019.

RUSSO, S. M. T. Espaço doméstico, devoção e arte: a construção histórica do acervo de oratórios brasileiro, séculos XVIII e XIX. Tese (Doutorado). São Paulo: FAUUSP, 2010.

SANTOS, M. O espaço do cidadão. 7. ed. São Paulo: EdUSP, 2007.

SENNETT, Richard. O Artífice. Rio de Janeiro: Editora Record, 2012.

SILVA, Giovani José da. Objetos biográficos de memória: reconhecendo-se como agentes históricos a partir de aulas de prática de ensino de história. Revista Textos FCC, São Paulo, v. 50, p. 1-148, nov. 2016. Disponível <http://publicacoes.fcc.org.br/ojs/index.php/textosfcc/article/view/4319/3235>. Acesso em: 10 mai. 2019.

TREINTA, F. T.; FARIAS FILHO, J. R.; SANT'ANNAC, A. P; RABELO, L. M. Metodologia de pesquisa bibliográfica com a utilização de método multicritério de apoio à decisão. Revista Produção, São Paulo, v.24, n.3, p.508-520, jul/set, 2014. 